\title{
Anregungen zur Qualitätssicherung in der 3-D-numerischen Modellierung mit FLOW-3D
}

\author{
FLOW-3D ist eine leistungsfähige 3-D-numerische Strömungssimulationssoftware für Frei- \\ spiegelabflüsse. Das strukturierte und entlang der Koordinatenachsen ausgerichtete Berech- \\ nungsnetz stellt eine Besonderheit der Software dar. Dadurch ergeben sich zusätzliche Frei- \\ heiten, wie die Implementierung von bewegten Objekten oder die vereinfachte Variations- \\ untersuchung von Einbauten. Im Hinblick auf die Qualitätssicherung muss die Adaptierung \\ der Geometrie an das Berechnungsnetz im Detail kontrolliert werden. Dies und weitere \\ Modellierungsaspekte werden in diesem Beitrag behandelt.
}

\section{Einleitung}

\subsection{Motivation}

Für die Bearbeitung von wasserbaulichen Fragestellungen werden neben dem klassischen skalierten Modellversuch im Labor vermehrt numerische Modelle herangezogen. Mit dem Einsatz der Numerik können Bandbreite, Flexibilität und Aussagekraft der möglichen Untersuchungen deutlich ausgeweitet werden. Anwendungsbeispiele numerischer Modelle reichen von großräumigen Geschiebebetrachtungen [12], [13] bis hin zur rein hydraulischen Optimierung einzelner Anlagenteile für die Wasserkraftnutzung [9], [15]. Die Errichtung eines physikalischen Modells ist in zeitlicher und finanzieller Hinsicht sehr aufwändig. Aus diesem Grund werden bei großen Modellversuchen vermehrt hybride Modellkonzepte gewählt [2], [10]. Durch die Kombination von physikalischem Modellversuch und numerischen Methoden können die Vorteile beider Modellansätze genutzt werden. In der Folge wird der zumeist personal- und kostenintensivere Laborversuch konzentriert in jenen Bereichen eingesetzt, in denen dieses Werkzeug optimale Ergebnisse liefern und nicht ersetzt werden kann.

Im wasserbaulichen Ingenieurbereich gehören 1-D- und auch 2-D-numerische Verfahren zu den Standardwerkzeugen. Die 3-D-Numerik ist (noch) eher an den Universitäten angesiedelt, doch der Bedarf an solchen Untersuchungen nimmt in der Praxis deutlich zu [6]. Die Möglichkeit, im frühen Planungsstadium mit verhältnismäßig geringem Mehraufwand das gewählte Design zumindest grob überprüfen zu können, ist dabei besonders von Vorteil. Um die Akzeptanz dieser Methoden noch weiter zu verbessern, werden im Zuge von Forschungsprojekten laufend Validierungsuntersuchungen durchgeführt und die Ergebnisse veröffentlicht [1], [3], [4], [9], [11], [15], [16], [17]. Zusätzlich gilt es, eine fundierte Qualitätssicherung zu verankern und die gewonnenen Erfahrungen aus der universitären Praxis mit anderen Anwendern und Auftraggebern zu teilen. Anhand des Beispiels der 3-D-numerischen Strömungssimulationssoftware FLOW-3D [7] werden nachfolgend die Besonderheiten sowie die Vor- und Nachteile dieses Softwareproduktes aufgezeigt. Zusätzlich wird ein Überblick über die an der Universität gesammelten Erfahrungen im Hinblick auf die Qualitätssicherung der Modellierung präsentiert. Ohne Anspruch auf Vollständigkeit sollen Anregungen für einen bestmöglichen Einsatz der Software vermittelt werden. Teilweise sind die beschriebenen Punkte abhängig von der verwendeten Version, da die Software laufend weiterentwickelt wird.

\subsection{FLOW-3D}

Die kommerzielle 3-D-numerische Strömungssimulation FLOW-3D bietet basierend auf einem strukturierten Berech- nungsgitter (Finite Differenzen) und dem Volume-of-Fluid-Verfahren (VOF-Verfahren) eine breite Palette an Simulationsmöglichkeiten [14]. Seit Jahren wird auch an der Universität Innsbruck und der EPFL Lausanne die Software in Lehre und Forschung eingesetzt. Durch die Stärken bei der Simulation von freien (Wasser-) Oberflächen (TruVOF-Technik [7]) ist FLOW-3D für wasserbauliche Anwendungen sehr gut geeignet. Für die Modellierung von reinen Rohrströmungen liefern alternative Softwarelösungen, wie zum Beispiel ANSYS-CFX, stabilere und bessere Ergebnisse [4], [9], [15]. Dies lässt sich zum einen durch die Beschränkung in der Gittererstellung begründen, und zum anderen neigt der Solver von FLOW3D ohne eine freie Oberfläche zu unphysikalischen Druckschwankungen. Eine weitere Schwäche der Software FLOW-3D stellt das relativ umständliche Post-Processing dar. Da aber eine umfassende Überarbeitung der Ergebnisauswertung vom Hersteller angekündigt ist (Stand Mitte 2013), wird auf diesen Punkt nicht gesondert eingegangen.

Das Hauptaugenmerk des Beitrages liegt auf der Besonderheit der Software FLOW-3D, die sich aus dem strukturierten Berechnungsnetz ergibt. Die importierte Geometrie wird automatisiert in der Software an das Netz angepasst. Die Simulation erfolgt mit der Berechnungsgeometrie, die sich doch deutlich von der ursprünglich importierten Geometrie unter- 
scheiden kann. Bei den meisten anderen 3-D-numerischen Software-Produkten wird hingegen das Netz an die Geometrie angepasst (Bild 1). Die Qualitätssicherung nach den allgemein gültigen Best-PracticeRichtlinien für numerische Berechnungen [5] muss somit für die Anwendung von FLOW-3D um spezifische Punkte ergänzt werden. Diese Aspekte werden detailliert beschrieben und mit einen Überblick über die Berechnungspraxis ergänzt. Ziel des Beitrages ist es, die in den letzten Jahren entwickelte und praktizierte Qualitätssicherung für FLOW-3D zusammenzufassen und anderen Nutzern zur Verfügung zu stellen.

\section{Geometrie des Berechnungsgebietes}

\subsection{Erstellung und Export}

Am Beginn einer Bearbeitung steht die Erstellung des 3-D-Modelles. Eine umfassende Qualitätssicherung setzt bereits bei der Beschaffung und Bewertung der Rohdaten an. Diese können bei einer bestehenden Geometrie von wenigen Punkten über (Bestands-) Pläne bis hin zu hochaufgelösten dreidimensionalen Laserscans reichen. Bei Neuplanungen stehen zumindest digitale 2-D-Pläne und vermehrt auch 3-D-Modelle zur Verfügung. Die Datendichte sollte möglichst in einem angemessenen Verhältnis zur Gitterauflösung des Berechnungsnetzes stehen.

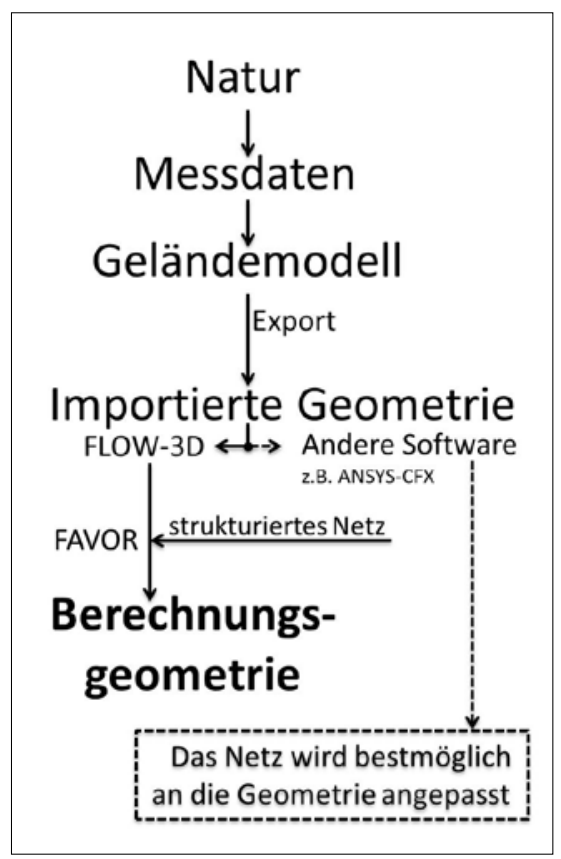

Bild 1: Prozesskette der Modellerstellung
Beispielhaft für eine zu untersuchende Geometrie und die Relevanz adäquater Vermessungsdaten wird eine bestehende Pegelmessstelle mit einer Schwelle unmittelbar unterstrom des Messquerschnittes herangezogen. Für das Bauwerk selbst existieren entsprechende Pläne, die aus der Planungsphase stammen. Die Schwelle hat einen deutlichen Einfluss auf die Schlüsselkurve des Pegels. Zusätzlich ist aber auch eine Beeinflussung der Pegelmessung durch die Lage der Sohle im ober- und unterwasserseitigen Bereich nicht außer Acht zu lassen [19]. Im besten Fall hat sich ein natürliches Gleichgewicht eingestellt und die Sohle ist (relativ) stabil. Eine gewisse Unsicherheit bei der Extrapolation der Schlüsselkurve bleibt aber immer erhalten, da insbesondere im Hochwasserfall diese Bereiche von Auflandung und Erosion betroffen sind.

Eine vermessungstechnische Aufnahme des Anströmungs- und Abströmungsbereiches ist in den meisten Fällen unumgänglich. Wünschenswert ist dabei eine flächige Aufnahme der Sohle und der Ufer. Eine solche flächige Aufnahme der Sohle wird in Zukunft mit Hilfe des grünen Lasers, der auch in Wasser bis zu einer gewissen Trübung und Tiefe eindringen kann [21], möglich sein. Dies bringt eine deutliche Vereinfachung der Vermessung und Erhöhung der Datendichte. Zudem stellt es eine kostengünstige Alternative für ein kontinuierliches Monitoring der Gewässersohle vor und nach dem Pegel dar.

Trockene Bereiche können mit den modernen motorisierten Theodoliten halbautomatisiert relativ einfach aufgenommen werden. Je nach Gewässertyp kann die terrestrische Aufnahme der benetzten Sohle mit deutlich unterschiedlichem Aufwand verbunden sein. Aus der Erfahrung kann als Richtwert für eine adäquate Abbildung der Gerinnestruktur circa ein Punkt pro Quadratmeter inklusive einer Verdichtung bei starken Veränderungen als Richtwert angegeben werden. Sehr hilfreich ist es, wenn im Vorfeld das direkte Gespräch mit dem Messtrupp gesucht wird und die Anforderungen der Modellierung besprochen werden. Zum Beispiel ist es zielführend, dass von der reinen Profilaufnahme abgegangen wird. Zusätzlich sollten große Steine im Bett nicht nur an deren höchsten Erhebung gemessen werden, sondern deren Umrisse mit mehreren Punkten aufgenommen werden. Dies vereinfacht die automatisierte Oberflächengenerierung und verhin- dert, dass der Stein als einzelner Punkt ins Oberflächenmodell eingeht und letztlich im Zuge der Netzgenerierung nicht abgebildet wird. Ein gewisses Maß an Nachmodellierung ist hier aber kaum zu umgehen.

Werden Bauwerke anhand von Plänen in das Modell integriert, ist neben der Lagerichtigkeit auch darauf zu achten, dass bei zwei oder mehr erstellten Volumenkörpern eine deutliche Überlappung eingeplant wird. Dadurch können Probleme vermieden werden, die durch die nachfolgend noch beschriebene Erstellung der Berechnungsgeometrie entstehen können.

FLOW-3D bietet generell drei Möglichkeiten der Geometrieverarbeitung: (a) Modellierung mit Hilfe einfacher Volumenkörper, (b) Import von Geländedaten als Punkte und (c) stl-Dateien. Für Sonderfälle können die beiden erstgenannten Varianten Vorteile mit sich bringen, doch wird im Regelfall wohl der Weg über ein CAD-Programm und damit über die Verwendung von stl-Dateien gewählt werden. Dabei handelt es sich um ein Dateiformat, welches Oberflächen als Dreiecksflächen beschreibt. Die im Bauwesen weit verbreitete Software AutoCAD ermöglicht den Export der stl-Dateien. Die damit verbundene Linearisierung von komplexen gekrümmten Geometrieteilen ist nur grob durch die Einstellung der Variablen FACETRES möglich. Im Gegensatz dazu bietet die Software Rhinoceros [8] eine genaue Einstellmöglichkeit der maximal zulässigen Abweichung zwischen dem ursprünglichen Modell und der exportierten Datei. Im Hinblick auf die umfassenden Möglichkeiten bei der 3-D-Modellierung ist Rhinoceros auch für die Erstellung des Berechnungsgebietes zu empfehlen.

\subsection{FAVOR}

Eine Besonderheit von FLOW-3D ist, dass das Berechnungsnetz aus kartesischen Gitterzellen besteht. Die Verwendung eines Rechengitters in Zylinderkoordinaten ist ebenfalls möglich. Krummlinige Berandungen der Geometrie werden durch das Blockieren (ganz oder teilweise) von Gitterzellen abgebildet. Dieses Vorgehen erleichtert dabei einerseits die Netzerstellung und beschleunigt andererseits den numerischen Lösungsprozess. Außerdem können dadurch bewegte Objekte [11] oder zusätzliche Einbauten für Untersuchungen von Varianten sehr einfach integriert werden, ohne dass jeweils das Berechnungsnetz grundlegend angepasst 


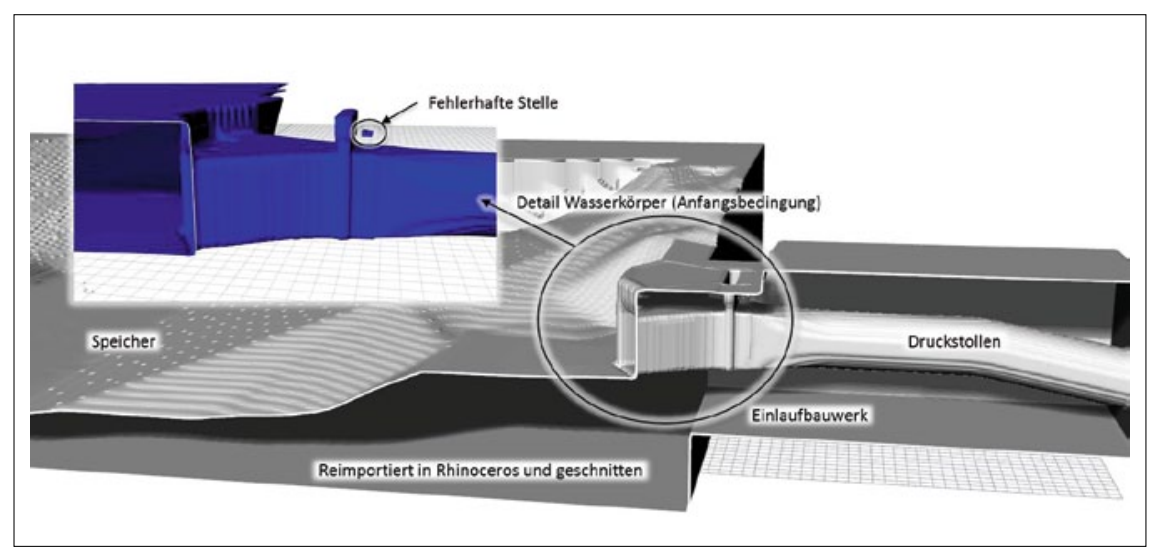

Bild 2: Geschnittene Berechnungsgeometrie nach Reimport in Rhinoceros

werden muss. Der Nachteil dieses Verfahrens ist, dass die reale Geometrie nicht genau abgebildet wird, sondern in Abhängigkeit von der Gitterauflösung besser oder schlechter angenähert wird (innerhalb einer Zelle gibt es nur einen linearen Verlauf der Geometrie, zweidimensional entspricht das Verfahren der stückweisen linearen Approximation einer Kurve). Damit ist neben der Messung, Modellierung und dem Export noch ein vierter Aspekt bei der Erzeugung der Berechnungsgeometrie, nämlich die Qualität der Diskretisierung, d. h. der Annäherung der realen Geometrie durch ganz oder teilweise blockierte Quader, zu beachten.

Die diskretisierte Geometrie ist als Vorschau in der Software selbst ersichtlich. Typischerweise kommt es zu Ausrundungen von Kanten und Abtreppungen von Flächen, die nicht entlang von Koordinatenebenen ausgerichtet sind. Im Zuge der Anzeige werden im Bearbeitungsordner auch entsprechende stl-Dateien exportiert (isofavor0000.stl und isofluid0000.stl für die Vorfüllung), die eine externe Kontrolle dieser Anpassung ermöglichen. Im Gegensatz zu AutoCAD kann Rhinoceros diese Datei wieder importieren, wodurch ein direkter Vergleich mit der ursprünglichen Geometrie und der Berechnungsgeometrie möglich wird (Bild 2). Dies erleichtert das Auffinden von fehlenden Geometrieteilen und Fehlstellen. Probleme können zum Beispiel auftreten, wenn aneinander anschließende Bauteile nicht überlappen oder Kanten in einer Zelle liegen. Der Algorithmus von FLOW-3D bildet unter gewissen Umständen kleine Hohlräume, die im Zuge der Berechnung zu Problemen führen können (Bild 2). Zusätzlich sollte besonderes Augenmerk auf Bauteile gerichtet werden, bei denen die Höhenlage für die Berechnung entscheidend ist. So könnte es bei der Pegelmessstelle (siehe oben) zu deutlichen Abweichungen bei der Schwelle kommen. Im schlechtesten Fall wird die Schwelle nur in der Achse terrestrisch aufgenommen und

\section{Springer Vieweg}

\section{Seit über 50 Jahren DAS anerkannte Standardwerk zur Wasserversorgung}

16., vollst. überarb. und akt. Aufl. 2014. XLII, 978 S. 365 Abb. Geb.

$€$ (D) $99,99|€(A) 102,79|{ }^{*}$ sFr 124,50

ISBN 978-3-8348-2560-5

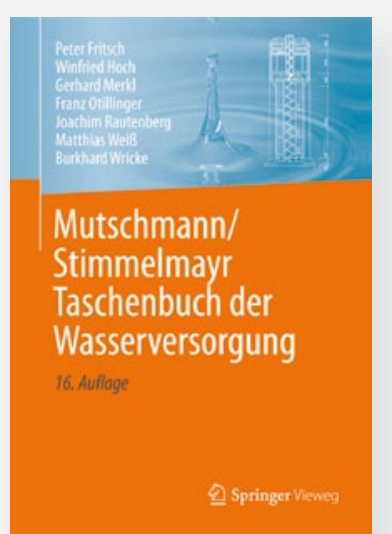

P. Fritsch, W. Hoch, G. Merkl, F. Otillinger, J. Rautenberg, M. Weiß, B. Wricke

Mutschmann/Stimmelmayr

Taschenbuch der Wasserversorgung

Das Taschenbuch der Wasserversorgung ist ein seit mehr als 50 Jahren anerkanntes, handliches Fachbuch, das alle Bereiche der Wasserversorgung umfasst. Dieses Buch begleitet als umfassendes, übersichtliches und unerlässliches Standardwerk in der Wasserversorgung tätige Ingenieure von ihrem Studium in den Beruf und durch die Karrierestufen hindurch. 
nicht gesondert modelliert. Die automatische Vernetzung erzeugt eine scharfe Kante und bei einem ungünstigen Netz (Knoten des Berechnungsgitters liegen nicht auf der Kante der Schwelle) sind je nach Auflösung des Berechnungsnetzes Abweichungen im Dezimeterbereich möglich. Es ist somit immer sinnvoll, die diskretisierte Geometrie während der Netzerstellung schon auf Vollständigkeit und Plausibilität zu überprüfen. Damit kann kontrolliert werden, ob feingliedrigerere Bauteile, wie Rechenstäbe, Drosseln oder vergleichbare Bauteile, generell von dem gewählten Berechnungsgitter aufgelöst werden können.

Eine wirkliche genaue Kontrolle ist nur sehr lokal und erst mit Hilfe von 2-DSchnitten in der Auswertung möglich. Dabei müssen die Grenzen der Anzeige schrittweise so verengt werden, dass die entsprechende Kante im Detail ersichtlich ist. Durch den visuellen Vergleich mit der Skala kann die tatsächliche Höhenlage bestimmt werden. Laut Auskunft des Softwaresupports gibt es keinen einfacheren Weg, um die tatsächliche Geometrie zu kontrollieren, die für die Berechnung herangezogen wird. Die Möglichkeit einer leichter handhabbaren Überprüfung sowie der Integration einer flächigen Kontrolle mit voller Genauigkeit ist derzeit eines der wesentlichen Anliegen der Anwender an den Softwarehersteller.

Die Verwendung eines feineren Rendering-Mesh, welches nur die optische Darstellung der Geometrie verbessert, wird dabei als nicht sinnvoll erachtet. Werden die ursprünglichen stl-Dateien im PostProcessing anstatt der tatsächlichen Berechnungsgeometrie eingeblendet, ist es unumgänglich, dies auch entsprechend $\mathrm{zu}$ dokumentieren und darauf hinzuweisen.

\section{Netzerstellung}

Das Berechnungsgitter besteht aus einem oder mehreren quaderförmigen Gitterblöcken mit einem strukturierten Gitter aus kartesischen Zellen. Eine bestmögliche Diskretisierung der Geometrie erreicht man, wenn man diese entlang der Koordi-

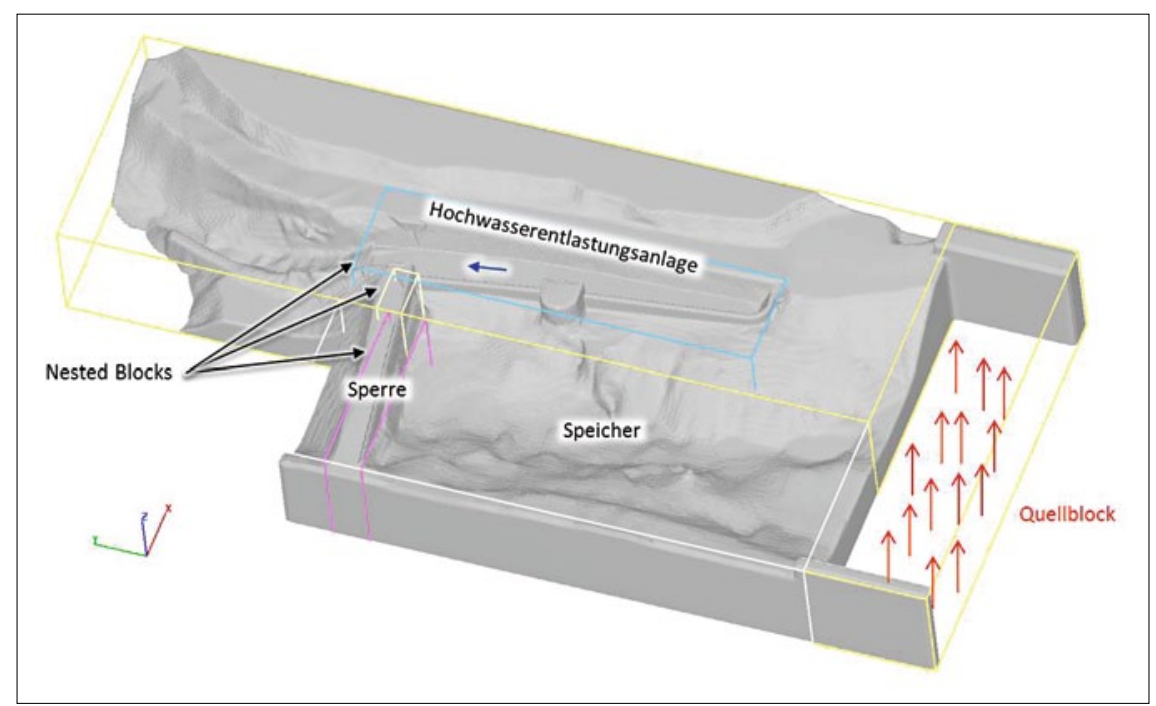

Bild 3: Ergänzte Vorschau der diskretisierten Geometrie in FLOW-3D [10]

natenachsen ausrichtet. Bei konstantem Gefälle erhält man ein besseres Rechengitter, wenn man die Geometrie waagrecht ausrichtet und das Gefälle durch einen der Neigung entsprechenden Schwerkraftvektor modelliert. Durch die Aneinanderreihung von mehreren Gitterblöcken (linked Multi-Block-Meshes) können auch komplexe Formen gut aufgelöst werden. Dabei ist aber zu beachten, dass es zu Rundungsfehlern an den Blockübergängen kommen kann. Um diese möglichst gering zu halten, sollten eher weniger Gitterblöcke verwendet werden. Zusätzlich sollte darauf geachtet werden, dass die Knoten bei Übergängen jeweils lagemäßig übereinstimmen. Eine Halbierung der Abstände zwischen den Knoten (Zellgrößenverhältnis) ist bei Wechseln der Auflösung zu bevorzugen. Dies gilt auch, wenn Blöcke in einen anderen Block eingefügt werden (nested Multi-Block-Meshes, Bild 3). Dadurch kann lokal das Berechnungsnetz verfeinert werden.

Die Erfahrung hat gezeigt, dass das Anschlagen von Wasser an die obere Grenze des Gitterblocks meist zu gravierenden Berechnungsfehlern führt. Entsprechend sollte der Abstand vom erwarteten Wasserspiegel im Berechnungsgebiet bis hin zur oberen horizontalen Grenze der Gitterblöcke an jeder Stelle im Berechnungsgebiet ausreichend groß gewählt werden. Alternativ könnte man das Rechengebiet am oberen Rand mit einer Druckrandbedingung öffnen und damit das Wasser bei zeitweisem Erreichen dieses Pegels ausströmen lassen. Um alle physikalischen Strömungsprozesse korrekt darstellen zu können, ist eine Mindestanzahl an durchströmten Zellen zwischen Wasseroberfläche und Wand erforderlich. Zum Beispiel sollte bei einem überströmten rundkronigen Wehr die untersuchte Wasserhöhe nicht kleiner als die Höhe von fünf Zellen sein [18]. Ist der Abstand geringer, besteht die Gefahr, dass die Annahmen der Grenzschicht die Strömung verfälschen. Zusätzlich können nicht benetzte oder durchströmte Bereiche durch Geometrieelemente aufgefüllt werden. Diese Bereiche werden in der Berechnung ausgeblendet. Im Lauf des Pre-Processings werden die Anzahl der aktiven Zellen und die Gesamtzahl der Zellen ausgegeben. Für die Berechnung sind nur die aktiven Zellen entscheidend, doch werden alle Zellen in der Lösungsdatei abgespeichert. Dies führt sehr schnell zu großen Ergebnisdateien, die aber durch Komprimieren (rar-Datei) relativ einfach um den Faktor 10 bis 100 reduziert werden können.

Es bestehen verschiedene Möglichkeiten zur Spezifikation des Rechengitters. Nach der Erfahrung der Autoren erzielt man die besten Ergebnisse, wenn man die

\section{Anzeigenschluss}


Anfangs- und Endkoordinaten der Blöcke in den drei Raumrichtungen angibt sowie die Anzahl der Gitterzellen in jeder Richtung. Dabei ist zu beachten, dass die Zellen ein bestimmtes Längen-/Breitenverhältnis (aspect ratio) nicht überschreiten. Es ist sinnvoll, sich vom am höchsten aufgelösten Bereich ausgehend nach außen zu arbeiten.

\section{Randbedingungen}

Die Definition von Randbedingungen ist in FLOW-3D nur an den Gitterblockgrenzen möglich und auch notwendig. Da die Software prinzipiell instationär rechnet, ist die zeitliche Anpassung der Eingabegrößen mit Hilfe von Tabellen relativ einfach. Für wasserbauliche Fragestellungen wird meist nur die Definition von Wasserspiegellagen und Abflüssen benötigt. Beim Beispiel der Bestimmung des Überfallbeiwertes [18], [19] kann eine der beiden Varianten als Oberwasserrandbedingung gewählt werden. Im einen Fall wird der Wasserspiegel und somit die Energielinie fixiert und der Durchfluss ermittelt. Wird der Abfluss vorgegeben, ergibt sich die Wasserspiegelhöhe. Welche Randbedingung gewählt wird, kann in Abhängigkeit von der genauen Fragestellung oder von der jeweiligen Genauigkeit der gemessenen Kalibrierungsdaten entschieden werden.

Die Modellierung eines fixierten $\mathrm{Ab}$ flusses ist entweder direkt über eine $\mathrm{Ab}$ fluss- bzw. Q-Randbedingung oder durch die Definition der Geschwindigkeit und der durchströmten Querschnittsfläche am Modellrand realisierbar. Die Q-Bedingung weist Vorteile bei nicht klar definierten Rändern auf, wobei mehr Rechenzeit benötigt wird, da jeweils zusätzlich ein Integral über die Einströmfläche gebildet und intern die Geschwindigkeit angepasst werden muss. Die Definition eines Quellblocks, d. h. die Vorgabe einer Einströmgeschwindigkeit (vertikal) von unten durch eine definierte Einströmfläche, die am oberstromigen Modellrand des Berechnungsgebietes über einen eigens definierten Gitterblock erfolgen kann, hat sich besonders bewährt. Das Wasser wird entsprechend vom Boden aus zugegeben, wodurch sich der Wasserspiegel unabhängig vom Typ der Randbedingung einstellt (Bild 3).

Wasserspiegellagen können mittels einer Druck-Randbedingung direkt angegeben werden. Vereinfacht wird dabei ein unendlich großer Speicher mit einem fixen Wasserspiegel simuliert. Mittels Definition der Wasserspiegelhöhe im Bereich der Sohle (aber noch innerhalb des Netzes) kann eine sehr stabile Ausflussrandbedingung modelliert werden. Kennt man an einem Auslass weder den Wasserspiegel noch den Abfluss, kann man eine Outflow-Randbedingung wählen. Diese sollte jedoch nur bei überkritischer Abströmung gewählt werden, da es ansonsten zu einem unphysikalischen Einstau kommen kann. Auf eine Kontrolle aller Flüsse über die Ränder (Massenbilanz) sollte nicht verzichtet werden.

\section{Weitere Aspekte der Modellierung}

Die Definition von Startwasser im Berechnungsgebiet im Sinne einer Anfangsbedingung kann entweder über die Eingabe von geometrischen Grenzen oder mit Hilfe von gesonderten stl-Dateien erfolgen. Diese Vorfüllung kann die notwendige Berechnungszeit deutlich verkürzen, wobei ein initiales Geschwindigkeitsfeld und die Option des hydrostatischen Druckes dies noch zusätzlich verbessern können. Die Verwendung von schon vorhandenen Berechnungsergebnissen (Restart) ist nicht zwingend vorteilhaft. Zum einen können Fehler in die neue Berechnung mit aufgenommen werden und zum anderen hat sich bei unterschiedlichen Modellen gezeigt, dass die Restart-Berechnung nicht zwangsläufig schneller ist als eine Neuberechnung.

Die Verwendung von Durchflussebenen (Baffles) und History-Points erleichtert die Auswertung von einzelnen Zielwerten der Numerik. Diese Werte können unter anderem für den Nachweis eines stationären Berechnungszustandes herangezogen werden.

Die Definition von Rauheiten ist im Allgemeinen individuell für jede Geometriegruppe möglich, die wiederum aus mehreren geladenen stl-Dateien bestehen kann. Dabei ist zu beachten, dass es sich um eine Rauheit handelt, die die Strömung in den Randzellen beeinflusst. Unter Umständen kann eine Verschiebung des Berechnungsnetzes zu unterschiedlich großen Randzellen führen, was wiederum einen Einfluss auf die berechnete Rauheit haben kann. Durch die Diskretisierung der Geometrie wird die geometrische Rauheit des Modells verändert. Eine Erhö- hung kann durch die abgetreppte Struktur von ursprünglich glatten Flächen, die nicht entlang der Koordinatenebene ausgerichtet sind, entstehen. Eine Verringerung der Rauheit kann aus der Abrundung von Kanten und Spitzen oder aus der nicht vollständigen Abbildung der Sohlenstruktur bedingt durch die Gittergröße resultieren. Eine Kalibrierung basierend auf Messdaten ist somit grundsätzlich obligatorisch. Sofern diese aufgrund nicht vorhandener Messdaten nicht durchführbar ist, kann im Sinne einer $\mathrm{Pa}$ rametervariation der Einfluss der gewählten Rauheit abgeschätzt werden.

Ein großer Vorteil der Software FLOW3D ist, dass die Wasserspiegellage relativ unabhängig von der gewählten Netzauflösung ist. Dies wurde mehrfach getestet und besonders bei größeren Wasserflächen konnte die Unabhängigkeit nachgewiesen werden. Die Ergebnisse können jedoch erhebliche Unsicherheiten aufweisen, sobald der Lufteintrag nicht mehr zu vernachlässigen ist. FLOW-3D bietet zwar die Möglichkeit, auch ein zweites Fluid und die Interaktion $\mathrm{zu}$ berechnen, doch besteht in diesem Bereich deutlicher Forschungsbedarf [20]. Weitere Untersuchungen sind auch bei dem nun zur Verfügung stehenden Sedimentmodell notwendig. Zwischenzeitlich konnten jedoch bereits gute Ergebnisse erzielt werden [3], [17].

\section{Danksagung}

Für die gute und konstruktive Zusammenarbeit richtet sich der Dank an die Herren Frieder Semler und Dr. Matthias Todte von Flow Science Deutschland $\mathrm{GmbH}$.

\section{Autoren}

\section{Dr. Roman Gabl \\ Dr. Bernhard Gems \\ Prof. Markus Aufleger}

Arbeitsbereich Wasserbau, Institut für Infrastruktur, Fakultät für Technische Wissenschaften Universität Innsbruck Technikerstraße 13 6020 Innsbruck, Österreich roman.gabl@uibk.ac.at bernhard.gems@uibk.ac.at markus.aufleger@uibk.ac.at

\section{Dr. Giovanni De Cesare}

École Polytechnique Fédérale de Lausanne, Laboratoire de Constructions Hydrauliques (EPFL-ENAC-LCH)

Station 18

1015 Lausanne, Schweiz

giovanni.decesare@epfl.ch 


\section{Literatur}

[1] Daneshvari, M.; Münch, C.; De Cesare, G.: Numerical simulation of a new sand trap flushing system. In: Proceedings of the 4th IAHR International Symposium on Hydraulic Structures, Porto, Portugal, 9.-11. Februar 2012.

[2] De Cesare, G.; Pfister, M.; Daneshvari, M.; Bieri, $M$.: Herausforderungen des heutigen wasserbaulichen Versuchswesens mit drei Beispielen. In: WasserWirtschaft 102 (2012), Nr. 7-8.

[3] De Cesare, G.; Daneshvari, M.; Federspiel, M.; Malquarti, M.; Epely Chauvin G.; Schleiss, A.: Étude sur modele physique et numerique des evacuateurs de crue et des fosses d'erosion du barrage de Koman en Albanie, In: La Houille Blanche (2011), Nr. 3, S. 48-55.

[4] Dobler, W.; Arch, A.; Larcher, M.; Zenz, G.: Numerische Untersuchung von Rohrströmungen am Beispiel eines Bifurkators. In: Österreichische Wasser- und Abfallwirtschaft 62 (2010), Nr. 3-4.

[5] ERCOFTAC: Best practice guidelines/ERCOFTAC Special Interest Group on Quality and Trust in Industrial CFD. Version 1.0. Casey, M.; Wintergerste, T. (Hrsg.): Special Interest Group on Quality and Trust in Industrial CFD. Brüssel:
European Research Community on Flow Turbulence and Combustion, 2000

[6] Faber, R.; Fuchs, M.; Puchner, G.: Numerische Simulation von Hochwässern: Von 1D zu 3D aus Anwendersicht im Ingenieurbereich. In: Österreichische Wasser- und Abfallwirtschaft 64 (2012), Nr. 5-6, S. 307-313.

[7] Flow Science Inc. (Hrsg.): FLOW-3D User's manual. Version 10.0, 2012)

[8] Gabl, R.: Rhinoceros als Unterstützung für das 3D-numerische Strömungssimulationsprogramm Flow-3D. In: Schleiss, A. (Hrsg.): 11. Treffen junger Wissenschafterinnen und Wissenschafter an Wasserbauinstituten. LCH Communication 40 (2009), S. 169-174.

[9] Gabl, R.: Numerische und physikalische Untersuchung des Verlustbeiwertes einer asymmetrischen Düse im Wasserschloss. In: Forum Umwelttechnik und Wasserbau. Innsbruck: innsbruck university press (IUP) (2012), Band 14.

[10] Gabl, R.; Achleitner, S.; Sendlhofer, A.; Höckner, T.; Schmitter, M.; Aufleger, M.: Optimierter Einsatz und Kombination von 3-D-Numerik und physikalischer Modellierung. In: WasserWirtschaft 103 (2013), Nr. 5, S. 128-131.

Roman Gabl, Bernhard Gems, Giovanni De Cesare and Markus Aufleger

\section{Contribution to Quality Standards for 3D-Numerical Simulations with FLOW-3D}

With the 3D-numerical simulation software FLOW-3D the modelling of free surface flow is user-friendly and reliable. This CFD software uses a structured rectangular volume mesh, which can be built up independent of the geometry. Therefore, variations of the geometry and moving objects are easy to model. But as a disadvantage, FLOW-3D has to reshape the imported geometry to fit it to the grid. This process is called FAVOR (Fractional Area Volume Obstacle Representation) method. The therewith connected characteristics and the needed adaptions of the general quality standards for numerical simulations are the main parts of the paper. In addition, several modelling issues are presented on the basis of the experiences with FLOW-3D.

Роман Габль, Бернхард Гемс, Джованни Де Чезаре и Маркус Ауфлегер

\section{Предложения по обеспечению качества в трехмерном численном моделировании с использованием FLOW-3D}

FLOW-3D - это эффективное трехмерное численное программное обеспечение для моделирования течения безнапорных стоков. Особенностью данного программного обеспечения является структурированная и ориентированная вдоль координатных осей расчетная сетка. Благодаря этому появляются дополнительные возможности такие как внедрение динамичных объектов или упрощенный вариационный анализ сооружений. Для обеспечения качества необходимо тщательно контролировать согласование геометрии и расчетной сетки. Эти и другие аспекты моделирования рассматриваются в данной статье.

[11] Gabl, R.; Kapeller, G.; Aufleger, M.: Lawineneinstoß in einen Speichersee - Vergleich numerisches und physikalisches Modell. In: WasserWirtschaft 100 (2010), Nr. 5, S. 26-29.

[12] Gems, B.: Entwicklung eines integrativen Konzeptes zur Modellierung hochwasserrelevanter Prozesse und Bewertung der Wirkung von Hochwasserschutzmaßnahmen in alpinen Talschaften. Modellanwendung auf Basis einer regionalen Betrachtungsebene am Beispiel des Ötztales in den Tiroler Alpen. In: Forum Umwelttechnik und Wasserbau. Innsbruck: innsbruck university press (IUP) (2012), Band 13.

[13] Habersack, H.; Hengl, M.; Huber, B.; Lalk, P.; Tritthart, M. et al.: Fließgewässermodellierung - Arbeitsbehelf Feststofftransport und Gewässermorphologie. Bundesministerium für Land- und Forstwirtschaft, Umwelt und Wasserwirtschaft \& Österreichischer Wasserund Abfallwirtschaftsverband (ÖWAV), Wien, 2011.

[14] Habersack, H.; Hengl, M.; Knoblauch, H.; Tritthart, M. et al.: Fließgewässermodellierung Arbeitsbehelf Hydrodynamik; Bundesministerium für Land- und Forstwirtschaft, Umwelt und Wasserwirtschaft \& Österreichischer Wasser- und Abfallwirtschaftsverband (ÖWAV), Wien, 2007.

[15] Huber, B.: Physikalischer Modelversuch und CFD-Simulation einer asymmetrischen Drossel in einem T-Abzweigstück. In: Österreichische Wasser- und Abfallwirtschaft 62 (2010), Nr. 3-4, S. 58-61.

[16] Mohringer, T.; Riester, J.; Kolb, S.; Nestmann, F.: Hybride Modelluntersuchung zum Ein- und Auslaufbauwerk im Unterbecken des Pumpspeicherkraftwerks Atdorf. In: WasserWirtschaft 103 (2013), Nr. 1-2, S. 72-77.

[17] Möller, G.; Boes, R.; Theiner, D.; Fankhauser, A.; Daneshvari, M.; De Cesare, G.; Schleiss, A.: Hybrid modeling of sediment management during drawdown of Räterichsboden reservoir. In: Schleiss, A.; Boes, R. (Hrsg.): Dams and Reservoirs under Changing Challenges. London: Taylor \& Francis Group, 2011.

[18] Pfister, M.; Battisacco, E.; De Cesare, G.; Schleiss, A. J.: Scale effects related to the rating curve of cylindrically crested Piano Key weirs. In: 2nd Intl. Workshop on Labyrinth and Piano Key Weirs (PKW 2013), Boca Raton: CRC Press, 2013.

[19] Plörer, M.; Gabl, R.; Aufleger, M.; Gattermayr, W.: Einfluss der Oberwassersohllage auf die Schlüsselkurve bei Pegelmessstellen an Wehren und Schwellen - physikalischer Modellversuch. In: Österreichische Wasser- und Abfallwirtschaft 65 (2013), Heft 3-4.

[20] Stamm, J.; Helbig, U.; Gierra, T.: Lufteintrag Eine Herausforderung bei physikalischen und numerischen Modellierungen. In: WasserWirtschaft 103 (2013), Nr. 5, S. 132-135.

[21] Steinbacher, F.; Pfennigbauer, M.; Ulrich, A.; Aufleger, M.: Airborne Hydromapping - die luftgestützte Kartierung von Flachwasserbereichen. In: WasserWirtschaft 99 (2009), Nr. 12, S. 10-14. 